\title{
The Lymphatic Drainage Pattern of Internal Mammary Sentinel Lymph Node Identified by Small Particle Radiotracer ( ${ }^{99 m}$ Tc-Dextran 40) in Breast
}

\author{
Xiao-Shan Cao, MD ${ }^{1,2}$ \\ Guo-Ren Yang, MD2,3 \\ Bin-Bin Cong, MD, $\mathrm{PhD}^{1,24}$ \\ Peng-Fei Qiu, MD1,2 \\ Yong-Sheng Wang, MD, PhD',2
}

${ }^{1}$ Department of Breast Cancer Center, Shandong Cancer Hospital Affiliated to Shandong University, Jinan,

${ }^{2}$ Shandong Academy of Medical Sciences, Jinan, ${ }^{3}$ Department of Nuclear Medicine, Shandong Cancer Hospital Affiliate to Shandong University, Jinan, ${ }^{4}$ School of Medicine and Life Sciences, University of Jinan and Shandong Academy of Medical Sciences, Jinan, China
Correspondence: Yong-Sheng Wang, MD, PhD Department of Breast Cancer Center,

Shandong Cancer Hospital Affiliated to

Shandong University, No. 440, Jiyan Rd, Jinan,

Shandong 250117, China

Tel: 86-53167626211

Fax: 86-53167626211

Email: wangysh2008@aliyun.com

Received January 24, 2018

Accepted June 7, 2018

Published Online June 11, 2018

\section{Purpose}

The purpose of this study was to detect the lymphatic drainage pattern of internal mammary area and verify the concept of internal mammary sentinel lymph node (IM-SLN) in breast.

\section{Materials and Methods}

A small particle radiotracer $\left({ }^{99 \mathrm{~m}} \mathrm{Tc}\right.$-Dextran 40$)$ was prepared and tested. ${ }^{99 \mathrm{~m}} \mathrm{Tc}$-Dextran 40 was injected into intraparenchyma at the sound breast by a modified radiotracer injection technique. Subsequently, dynamic single-photon emission computed tomography (SPECT), computed tomography (CT), and SPECT/CT combination images were performed to identify the radioactive lymph vessels and internal mammary lymph nodes (IMLNs). The direction of lymph drainage and the location of the IMLNs were identified in the SPECT/CT imaging.

\section{Results}

The radiochemical purity of ${ }^{99 m} \mathrm{mc}$-Dextran 40 was > 95\%. ${ }^{99 m} \mathrm{Tc}$-Dextran 40 could drainage into first, second, and third lymph node and the radioactive lymph node could be detected by the $\mathrm{y}$ detector in the animal experiment. After ${ }^{99 \mathrm{~m}} \mathrm{Tc}$-Dextran 40 injecting into intraparenchyma, 50.0\% cases (15/30) were identified the drainage lymphatic vessels and radioactive IMLNs by SPECT. The drainage lymphatic vessel was found from injection point to the first IMLN (IM-SLN) after 10.5 \pm 0.35 minutes radiotracer injection, and then ${ }^{99 m}$ Tc-Dextran 40 was accumulated into the IM-SLN. The combination imaging of SPECT/CT showed the second IMLN received the lymph drainage from the IM-SLN. The lymphatic drainage was step by step in the internal mammary area.

\section{Conclusion}

The lymph was identified to drain from different regions of the breast to IM-SLN, and then outward from IM-SLN to other IMLN consecutively. It demonstrated the concept of the IM-SLN and provided more evidences for the application of internal mammary sentinel lymph node biopsy.

\section{Introduction}

The status of internal mammary lymph nodes (IMLNs) plays an important role in completing staging, guiding clinical treatment, and judging prognosis as axillary lymph node (ALN) for breast cancer [1-3]. Until now, internal mammary lymph node irradiation (IMLNI) is based on the high risk of

\section{Key words}

Breast neoplasms, Lymph drainage,

Internal mammary sentinel lymph node, ${ }^{99 m}$ Tc-Dextran 40, SPECT/CT 
(IM-SLN) with high safety and feasibility $[7,8]$. Due to the accuracy of IM-SLN has not been validated and the low visualization rate of IM-SLN has not been improved, the application of IM-SLNB is restricted and the development of IM-SLNB is far behind axillary sentinel lymph node biopsy (ASLNB).

The accuracy of the axillary sentinel lymph node (ASLN) and its lymphatic drainage pattern has been validated by axillary lymph nodes dissection (ALND) [9-11]. However, IMLNs dissection has been abandoned in that it could not improve the survival of patients [12]. Thus, the accuracy of IM-SLN could not been proved by this method. As IMLN could receive drainage from all quadrants of tumors [13] and almost only intraparenchymal lymph flowing to the internal mammary region $[14,15]$, so we hypothesized that IM-SLN could receive lymphatic drainage from not only the primary tumor area but also the entire breast parenchyma. Based on this hypothesis, a modified radiotracer injection technique (periareolar intraparenchymal, high volume, and ultrasound guidance) was established, which improves the visualization rate of IM-SLN significantly and helps to promote the research of IM-SLNB [14].

Based on the modified injection technique, a small particle size colloid radiotracer ${ }^{99 \mathrm{~m}} \mathrm{Tc}$-Dextran $40,<30 \mathrm{~nm}$, molecular weight $40 \mathrm{kD}$ ), which could move to the secondary lymph nodes, was injected into the parenchyma and observed the lymphatic drainage pattern in the breast by lymphoscintigraphy of single-photon emission computed tomography/ computed tomography (SPECT/CT) to reflect the real lymphatic drainage of the breast from the aspects of the anatomy and physiology, and to provide more evidences to verify the concept of IM-SLN in this study.

\section{Materials and Methods}

The freeze-dried kit of Dextran was procured from Shihong Pharmaceutical Center (25 mg Dextran 40 [40 kD] and $0.25 \mathrm{mg} \mathrm{SnCl} 2$; Beijing, China). The $\mathrm{Na}^{99 \mathrm{~m}} \mathrm{TcO} 4$ was provided by the Department of Nuclear Medicine, Shandong Cancer Hospital Affiliated to Shandong University (Jinan, China). The ${ }^{99 \mathrm{~m}} \mathrm{Tc}$-Sulfur colloid, which is the common radiotracer for sentinel lymph node (SLN) biopsy in the clinical practice, was also provided by the Department of Nuclear Medicine. The methylene blue injection was procured from Jichuan Pharmaceutical Group Co., Ltd. (2 mL; Taixing, China). All chemicals and reagents were of analytical grade.

\section{Radiolabeling Dextran 40}

The freeze-dried kit of Dextran was solubilized with $\mathrm{Na}^{99 \mathrm{~m} T \mathrm{TO}} 4$ (1-2 mL, 370-740 MBq) to radiolabel the Dextran 40. The reaction mixture was fully shaken and then rest for 15 minutes at room temperature. In the clinical practice, the radiochemical purity of the radiotracer must be greater than $90 \%$ tested by radio-thin layer chromatography (TLC).
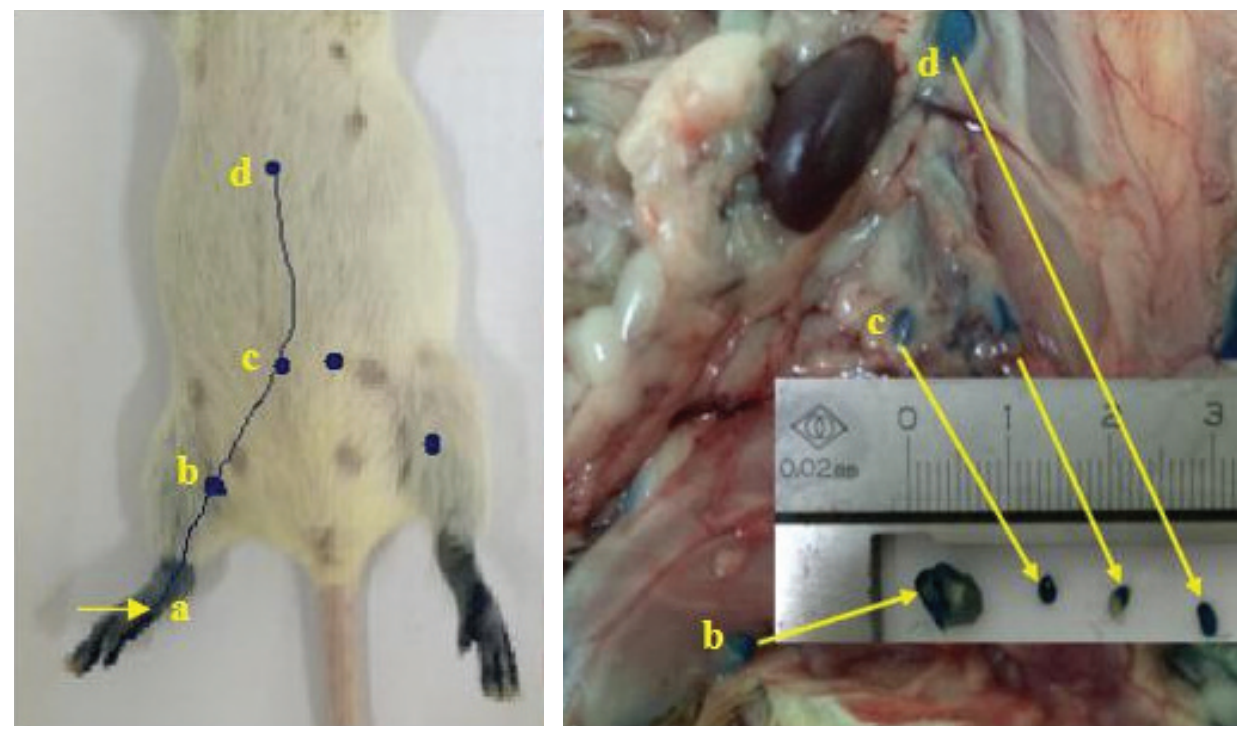

Fig. 1. The lymphatic drainage identified by blue dye in the hind foot of the mice. a, injection point; $b$, sentinel lymph node (popliteal); c, secondary lymph node (iliac); d, tertiary lymph node (renal). 


\section{Animal experiment}

Twenty-eight-week-old female BALB / c mice were ordered from Shandong University Laboratory Animal Center (Jinan, China). They were bred under specific pathogen-free conditions in the Basic Laboratory, Shandong Cancer Hospital Affiliated to Shandong University for 1 week. All mice were randomly divided into four groups (group 1, 2, 3, and 4), with six mice in each. After anesthesia with isoflurane, ${ }^{99 \mathrm{~m} T c-D e x t r a n} 40$ was injected subcutaneously into one hind footpad of mice of the experimental groups (group 1, $25 \mu \mathrm{Ci}$; group 2, $50 \mu \mathrm{Ci}$; and group 3, $250 \mu \mathrm{Ci}$ ). ${ }^{99 \mathrm{~m}} \mathrm{Tc}$-sulfur colloid ( $50 \mu \mathrm{Ci}$, boiling 5 minutes) was injected subcutaneously into one hind footpad of mice in the control group (group 4). The injection point, background, SLN (popliteal), secondary lymph node (iliac), tertiary lymph node (renal), and contralateral SLN (popliteal) were detected by $\gamma$ detector on the surface of skin to identify in vivo radionuclides counts. Each group was randomly separated into two subgroups. Subgroup A was observed at 2 hours continuously after injection and subgroup B was observed for 12 hours before sacrifice. Furthermore, methylthioninium chloride injection $(50 \mu \mathrm{L})$ was injected into all hind footpads 30 minutes before sacrifice.

The mice were sacrificed via cervical dislocation and subjected to dissection, following blue vital dye lymph vessel draining from the injection point to the blue dyed lymph nodes (Fig. 1). The injection point, background, popliteal lymph node, iliac lymph node, renal lymph node, contralateral popliteal lymph node, heart, liver, spleen, lung, and kidney were collected and ex vivo radionuclide counts were identified by a handheld $\gamma$ detector (Neoprobe 2000, Johnson \& Johnson, Dublin, OH).

\section{Clinical experiment}

\section{1) Enrollment of patients}

From December 2015 to July 2017, patients with histologically proven clinical stage $\mathrm{cT}_{1-4} \mathrm{~N}_{0-3} \mathrm{M}_{0}$ unilateral primary breast cancer treated at our breast cancer center were enrolled. Patients with inflammatory breast cancer, radiotherapy, prior ASLNB, or with a history of prior contralateral breast surgery were excluded.

\section{2) Injection of radiotracers}

All the patients received radio-labeled tracer injection intraparenchymally at the 6 and 12 o'clock positions 2.0-3.0 $\mathrm{cm}$ away from the nipple under ultrasonographic guidance 16-18 hours before surgery [14]. ${ }^{99 \mathrm{~m}} \mathrm{Tc}-$ labeled sulfur colloid (1.0-1.2 mL, 18.5-37 MBq) was injected in the breast with

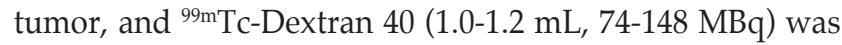
injected into the healthy breast.

\section{3) Dynamic lymphoscintigraphy}

Planar dynamic single-photon emission computed tomography (SPECT; Philips BrightView XCT, Philips Healthcare, Best, The Netherlands) scintigrphatic images were obtained 40-60 minutes (40-60 frames, 60 seconds per frame) in both anterior and lateral projection focusing on the area of interest subsequently after radiotracers injection to detect the direction of the lymphatic drainage. The SPECT acquisition conditions were set as matrix, $128 \times 128$; zoom, 1 ; peak energy, $140 \mathrm{keV}$; window width, $20 \%$. The direction of lymphatic channel could be identified on the monitor. The first "hot spot" on the lymphoscintigraphy, either in the internal mammary area or in the axilla area, was considered to be a SLN. The localization and the number of all visible focal accumulations of radioactivity were recorded to observe the lymphatic drainage pattern in breast.

\section{4) SPECT/CT combination imaging}

SPECT/CT was performed immediately after SPECT dynamic lymphscintigrapy to identify the location of ALNs and IMLNs. The SPECT / CT system consisted of a dual-head variable-angle gamma camera equipped with low-energy high-resolution collimators and a three-slice spiral CT scanner (120 kV, $20 \mathrm{mAs}, 4 \mathrm{~mm}$ slices) optimized for rapid rotation. The CT scanning was low-dose and non-contrast in this study. SPECT acquisition (matrix $64 \times 64$, zoom 1.46×[40.9] $\mathrm{cm}, 140 \mathrm{keV}$ ) was performed using steps of 6 degree (10 seconds per view). Maximum intensity projections with a threedimensional display were generated to localize SLNs in relation to anatomical structures. In addition, the SPECT/CT combination images were acquired again in the next day morning (about 1 hour before surgery) for about 11 minutes. In the tumor side, IM-SLN was detected by both the SPECT / $\mathrm{CT}$ and $\gamma$ detector, patients with radioactive IM-SLN received IM-SLNB. All enrolled patients were followed up 1 month to observe the complications.

\section{Data analysis}

This was a descriptive study with an analysis of the frequency of the findings. The data were analyzed with the SPSS ver. 17.0 software package (SPSS Inc., Chicago, IL). Pearson chi-square test or Fisher exact test was used for categorical variables. All tests were two-sided, and $\mathrm{p}<0.05$ was considered statistically significant. 
Table 1. The ex vivo radionuclides counts of each-tier lymph nodes in subgroup A ( $\mathrm{n}=12)$

\begin{tabular}{|c|c|c|c|c|c|c|}
\hline Group & $\begin{array}{l}\text { Radiotracer } \\
\text { doses (uCi) }\end{array}$ & SLN & $\begin{array}{l}\text { Second } \\
\text { LN }\end{array}$ & $\begin{array}{l}\text { Third } \\
\text { LN }\end{array}$ & $\begin{array}{l}\text { Contra-lateral } \\
\text { SLN }\end{array}$ & Background \\
\hline \multicolumn{7}{|c|}{ Experiment } \\
\hline 1 & 25 & $120 \pm 1.5$ & $56 \pm 1.6$ & $48 \pm 2.6$ & $10 \pm 1.7$ & $8 \pm 1.2$ \\
\hline 2 & 50 & $620 \pm 1.2$ & $129 \pm 1.7$ & $186 \pm 1.9$ & $16 \pm 1.2$ & $15 \pm 1.5$ \\
\hline 3 & 150 & $1,032 \pm 1.3$ & $424 \pm 1.5$ & $402 \pm 1.5$ & $48 \pm 1.2$ & $27 \pm 1.3$ \\
\hline \multicolumn{7}{|l|}{ Control } \\
\hline 4 & 50 & $550 \pm 1.1$ & 0 & 0 & 0 & $1 \pm 1.0$ \\
\hline
\end{tabular}

SLN, sentinel lymph node; LN, lymph node.

Table 2. Clinical characteristics of the patients with (+) and without (-) IMLN imaging in the experimental side

\begin{tabular}{|c|c|c|c|}
\hline Characteristic & IMLN- & IMLN+ & p-value \\
\hline \multicolumn{4}{|l|}{ Age (yr) } \\
\hline Median & \multicolumn{2}{|c|}{49.5} & \\
\hline$\leq 50$ & 7 & 12 & 0.128 \\
\hline$>50$ & 8 & 3 & \\
\hline \multicolumn{4}{|l|}{ BMI $\left(\mathrm{kg} / \mathrm{m}^{2}\right)$} \\
\hline $18.5-24.9$ & 11 & 12 & 1.000 \\
\hline $25.0-28.0$ & 4 & 3 & \\
\hline$>28.0$ & 0 & 0 & \\
\hline \multicolumn{4}{|c|}{ Mammographic density } \\
\hline ACR 1 & 6 & 1 & 0.074 \\
\hline ACR 2 & 6 & 10 & \\
\hline ACR 3 & 2 & 4 & \\
\hline ACR 4 & 1 & 0 & \\
\hline
\end{tabular}

IMLN, internal mammary lymph node; BMI, body mass index; ACR, American College of Radiology (ACR 1; almost entirely fat, glandular tissue < 25\%; ACR 2, scattered fibroglandular densities [ca. 25-50\% of breast]; ACR 3, heterogeneously breast dense [ca. $51-75 \%$ of breast]; and ACR 4, extremely dense [ $>75 \%$ of breast]).

\section{Ethical statement}

The study has been approved by the Shandong Cancer Hospital Affiliated to Shandong University Ethics Committee (No. SDTHEC20130324) and all subjects signed an informed consent form.

The animal experiment has been approved by the Shandong Cancer Hospital Affiliated to Shandong University Animal Ethics Committee (No. SDTHEC20130324).

\section{Results}

\section{Synthesis of ${ }^{99 \mathrm{~m}} \mathrm{Tc}$-Dextran 40}

The radiolabeling yield was $>95 \%$ using the freeze-dried kit of Dextran and $\mathrm{Na}^{99 \mathrm{~m}} \mathrm{TcO} 4$. The results of TLC analysis showed that $R$ value $0-0.1,0.3$ for ${ }^{99 m} \mathrm{Tc}-D e x t r a n ~ 40, \mathrm{Na}^{99 \mathrm{~m} T \mathrm{~T}-}$ O4, respectively.

\section{Animal experiment}

The model of lymphatic drainage in the hind leg was shown in the Fig. 1. In the experimental groups, the sentinel (popliteal) lymph node, the secondary (iliac) lymph node, 

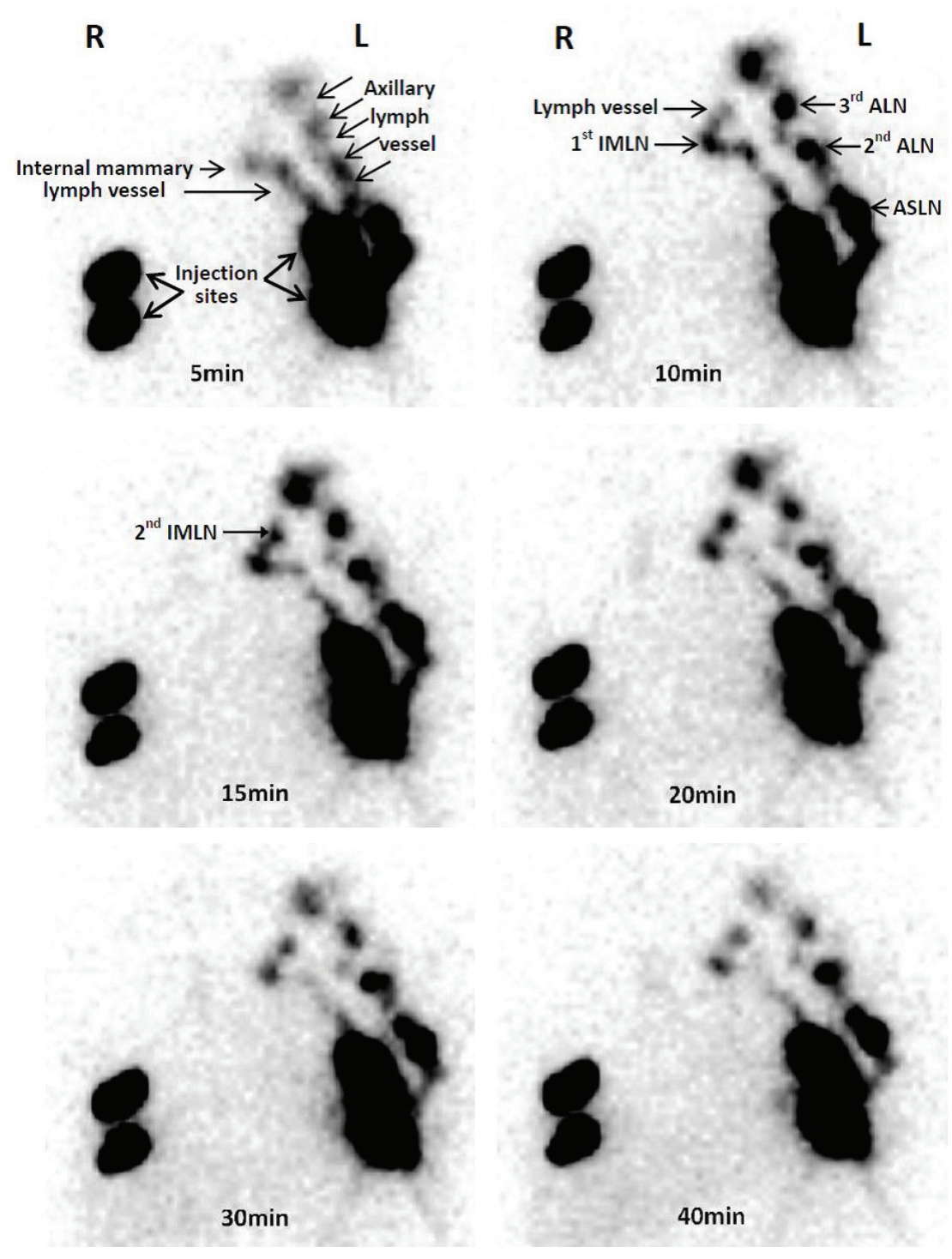

Fig. 2. The single-photon emission computed tomography images of dynamic lymphoscintigraphy. The small particle radiotracer could be identified in the lymphoscintigraphy with the time going. $R$, right breast is the tumor side; $L$, left breast is the sound side; IMLN, internal mammary lymph node; ALN, axillary lymph node; ASLN, axillary sentinel lymph node.

and the tertiary (renal) lymph node was detected by $\gamma$ detector at $5 \pm 0.59$ minutes, $6 \pm 0.64$ minutes, and $6.5 \pm 0.55$ minutes, respectively after ${ }^{99 \mathrm{~m}} \mathrm{Tc}-\mathrm{Dextran} 40$ injection. In subgroup A, the stained first, second, and third nodes removed could be detected ex vivo radionuclide counts after dissection (Table 1). The count of contralateral popliteal lymph node was nearly equal to the background. The first, second, and third nodes in subgroup B also could be detected in vivo radionuclide counts after 12-hour injection. The radionuclide counts increased with the increasing of radiation dose injection. In vivo radionuclide counts of kidney and liver detected in all experimental groups were higher than spleen and lung, and the counts were increasing as time going on, which indicated that the ${ }^{99 \mathrm{~m}} \mathrm{Tc}-$ Dextran 40 was metabolized by kidney and liver in mice. In the control group, ex vivo radionuclide counts were only detected in the sentinel (popliteal) lymph node (Table 1). Thus, ${ }^{99 \mathrm{~m}} \mathrm{Tc}$-Dextran 40 could enter each-tier lymph nodes detected by the $\gamma$ probe and show the whole lymphatic drainage pattern of the hind leg in mouse. 


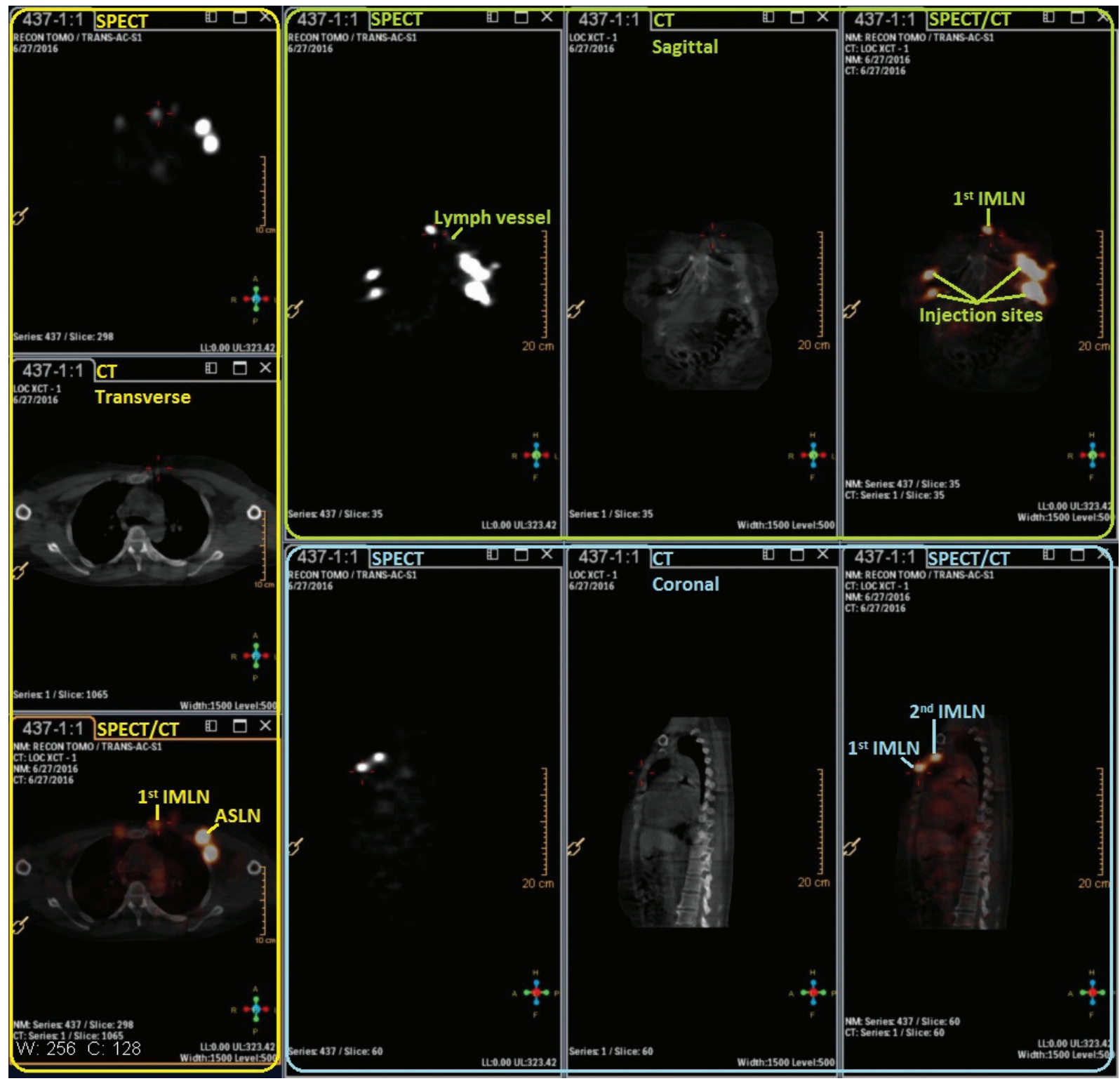

Fig. 3. The combination images of single-photon emission computed tomography (SPECT)/ computed tomography (CT) which helps to identify the intercostal location of internal mammary lymph nodes (IMLNs) from different anatomical aspects. ASLN, axillary sentinel lymph node.

\section{Clinical experiment}

\section{1) Patient characteristics}

A total of 30 patients with $\mathrm{cT}_{1-4} \mathrm{~N}_{0-3} \mathrm{M}_{0}$ breast cancer treated in our breast cancer center were enrolled into this study, and the median age was 49.5 years (range, 34 to 65 years). All of the demographic and clinicopathologic characteristics were listed in Table 2.

\section{2) Lymphatic drainage imaging in human body}

In the breast with ${ }^{99 \mathrm{~m}} \mathrm{Tc}$-Dextran 40 injection, the imaging of drainage lymph vessels could be detected by SPECT $4.5 \pm 0.12$ minutes after injection. It showed that the small radiotracer could drain to the lymph vessels from the injection site to the area of axilla or internal mammary. There were no any complications and reactions after 1-month follow-up.

In the area of axilla, the visualization rate of ALN was $90 \%$ 


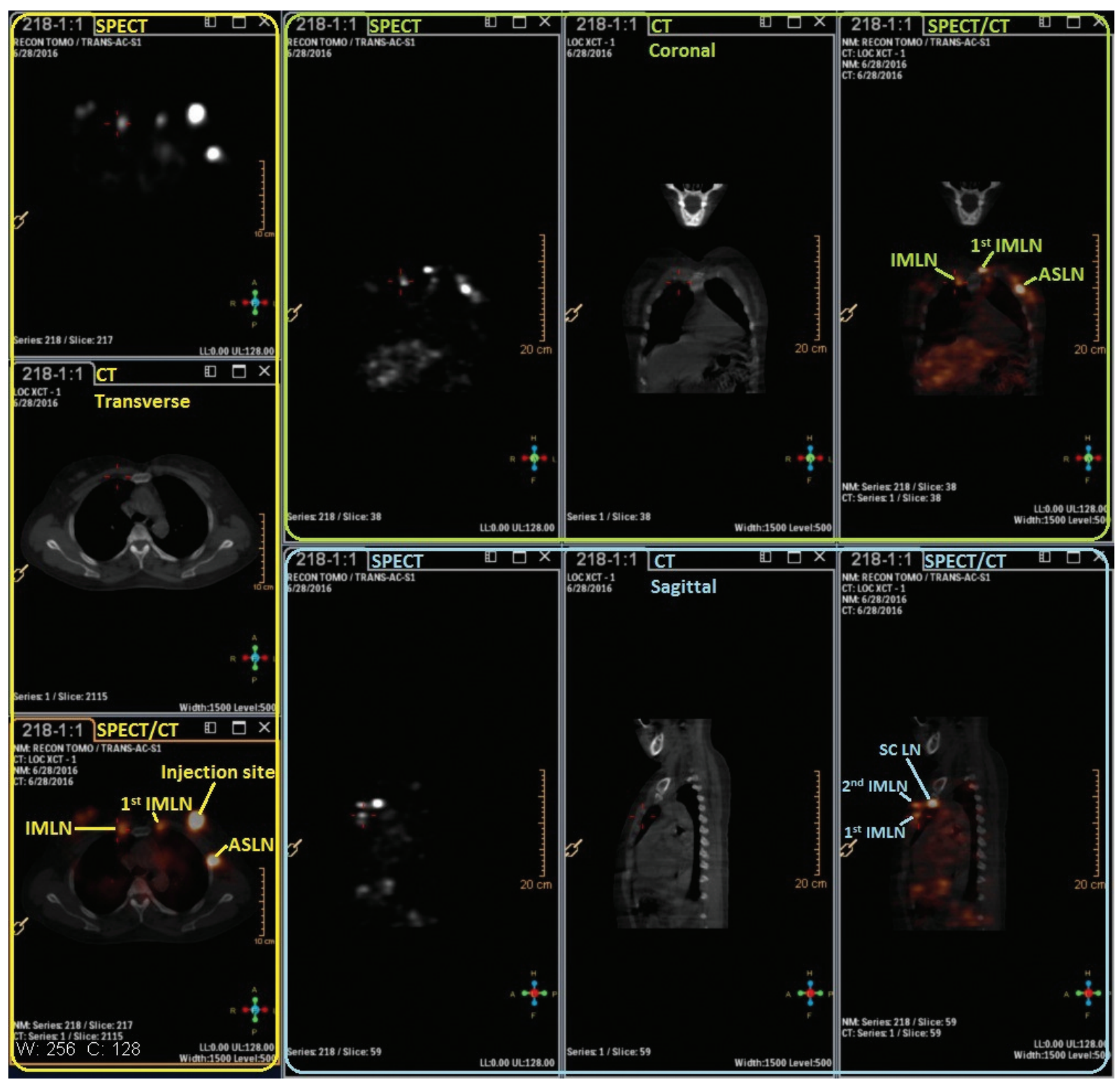

Fig. 4. The lymphoscintigraphy detected by the single-photon emission computed tomography (SPECT)/computed tomography $(\mathrm{CT})$ in the next day morning about 1 hour before surgery. IMLN, internal mammary lymph node; ASLN, axillary sentinel lymph node; SCLN, supraclavicular lymph node.

(27/30). The imaging of the first ALN (ASLN) could be detected by SPECT at $5.5 \pm 0.17$ minutes after injection, and then the small tracer drained from ASLN to the second-tier ALN at $6.5 \pm 0.12$ minutes after injection. The imaging showed that the lymphatic drainage was one-by-one from ASLN to the other ALNs (Figs. 2-4).

In the area of internal mammary, the visualization rate of IMLNs was 50\% (15/30). The first IMLN (IM-SLN) could be detected in the first to fourth intercostal, and the proportion of them was $6.7 \%(1 / 15), 33.3 \%$ (5/15), $46.7 \%$ (7/15), and
$13.3 \%$ (2/15), respectively, and only one lymph vessel could be detected draining to the IM-SLN. The imaging of the IM-SLN could be detected by SPECT at $10.5 \pm 0.35$ minutes after injection, and the second-tier IMLN could be detected from the IM-SLN at 15.4 \pm 0.21 minutes after injection. The lymph drainage was step by step in the IMLN chain (Figs. 2-4).

In the breast with ${ }^{99 \mathrm{~m}} \mathrm{Tc}$ - labeled sulfur colloid injection, the visualization rate of IM-SLN was $23.3 \%$ (7/30). The success rate of IM-SLNB was $100 \%(7 / 7)$. The median number of IM-SLNs was 2 (total, 10; range, 1 to 3). The site of IM-SLNs 


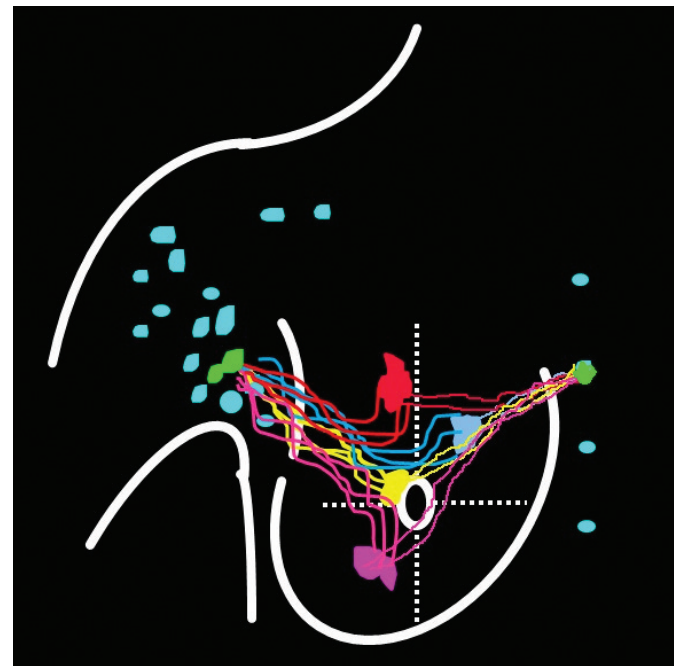

Fig. 5. The lymph in different area drain into the same internal mammary lymph node (IMLN), and then other IMLNs receive lymph from it one by one.

concentrated in the second $(40.0 \%, 4 / 10)$ and third $(40.0 \%$, $4 / 10)$ intercostal space. The median time-consuming of IM-SLNB was 10 minutes. The IM-SLN positive rate was $14.3 \%$ (1/7), combining with ALNs metastases.

\section{Discussion}

The study of the lymphatic drainage in the breast found that the ipsilateral ALNs receive more than $75 \%$ of the breast lymph and ipsilateral IMLNs receive other lymph [15]. As the same with ALNs, IMLNs could receive lymphatic drainage from all quadrants of the breast including primary tumor area [13].

In the IMLN chain, there might be two types lymphatics drainage pattern: one is the lymph in different area of the breast drain into the same IMLN, then other IMLNs receive the lymph from it one by one (Fig. 5); another is the lymph in different area of the breast draining into different IMLNs (Fig. 6). Only the IMLN lymphatic drainage pattern accords with the former one, the concept of IM-SLN is accuracy, and the pathology status of IM-SLN could reflect the situation of the whole IMLN chain. If the IMLN lymphatic drainage pattern accords with the later one, the pathology status of IM-SLN could not reflect the situation of the whole IMLN chain.

Due to extend radical mastectomy (including IMLNs dissection) had been abandoned as it did not improve survival

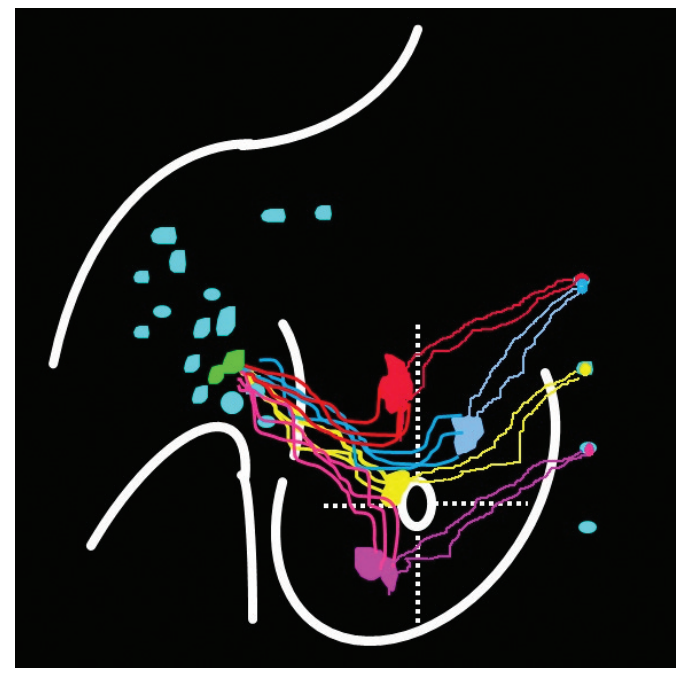

Fig. 6. The lymph in different area of breast drain into different internal mammary lymph nodes.

versus radical mastectomy in 1980s [12], the lymphatic drainage pattern of internal mammary area cannot be validated by this method. In 1786, Cruikshank and colleagues used the mercury to explore the lymphatics drainage in the breast $[16,17]$. In 1903, Poirer and Cuneo discovered the anatomy of the lymphatic system in the breast by using oil painting dye to stain the lymphatics [18]. However, the lymphatics of breast found by these methods were identified in the cadaver and it did not describe the IMLN lymphatics drainage pattern in details. Suami used hydrogen peroxide to identify the lymph vessels which injected with a lead oxide mixture and radiographed to study the IMLN lymphatics drainage pattern [19], but the result was also identified in the cadaver and could not reflect the really lymphatics drainage pattern in human body. With the development of imaging technique, Turner-Warwick injected radioisotope gold $\left(\mathrm{Au}^{198}\right)$ into tumor during surgery in breast cancer patients and found that both ALNs and IMLNs could receive lymphatic drainage from all quadrants of the breast [13]. While, due to the limitation of the tomography technology, dynamic lymphscintigrapy could not be applied and the IMLN lymphatic drainage pattern was not identified in this study. However, this study provided some important information for designing our study.

To explore the IMLN lymphatic drainage pattern and validate the accurate of the IM-SLN concept, one radiotracer with small particle size was injected into intra-parenchyma, and then performing dynamic lymphoscintigraphy to identify the intercostal location and the lymphatic drainage pattern of IMLNs. In our clinical experience, the vessel is visua- 
lized less often with ${ }^{99 \mathrm{~m}} \mathrm{Tc}$-labeled sulfur colloid. Colloids with different particles size (e.g., unfiltered sulfur colloid, 50-1,000 nm) enter the lymphatic vessels slowly through pinocytosis; even larger particles do not migrate from the injection site [20]. Smaller particles (e.g., size $<5 \mathrm{~nm}$ ) could easily leak into the bloodstream directly [21]. However, particles size between $5 \mathrm{~nm}$ and $25 \mathrm{~nm}$ could enter lymphatic capillaries via the gaps between cell junctions and the intercellular clefts formed by overlapping cells, which often allows us to visualize the lymphatic drainage vessels leading directly to the lymph node in the lymphoscintigraphy [20]. ${ }^{99 \mathrm{~m} T c-D e x t r a n} 40(<30 \mathrm{~nm})$ is a small particle colloid which has been proved in mice model. Based on the lymphatic drainage in the hind foot of mouse described by Harrell et al. [22], all blue dyed lymph nodes were caught by following the blue dyed draining lymph vessel. All dissected blue dyed lymph nodes have a high ex vivo radionuclide counts and the counts of second-tier lymph nodes were higher than $10 \%$ of the SLN. Thus, ${ }^{99 \mathrm{~m} T \mathrm{Tc}-D e x t r a n} 40$ could drain into lymphatic vessels and make every tier lymph nodes imaging in the hind-foot of mouse.

The lymphoscintigraphy technique plays an important role in SLN biopsy and could show the lymphatic drainage vessels passing from the tumor site to the draining lymph node after radiotracer injection [23]. It could find an unequivocal channel entering the first lymph node (defined as SLN) in the SPECT dynamic lymphoscintigraphy. SPECT/CT camera fuses tomographic lymphoscintigrams with anatomical data from CT [24]. The combination images of SPECT/CT provide functional and anatomical images in the same scanning session and help to identify the intercostal location of IMLN (Figs. 3 and 4). SPECT / CT provides an anatomical overview in three-dimensional perspectives that cannot be provided by planar images or intraoperative lymphatic mapping techniques $[25,26]$.

The accuracy of ASLN has been validated by ALND [9-11]. The results of our study showed that the second-tier ALN received the lymphatic drainage vessel from the ASLN, which accord with the concept of ASLN (Fig. 2). It indicated the accuracy of our method to identify the IMLN lymphatic drainage indirectly.

In our study, lymphatic vessel draining to internal mammary area could be detected by SPECT at $4.5 \pm 0.12$ minutes after the ${ }^{99 \mathrm{~m} T \mathrm{Tc}-D e x t r a n} 40$ injected intraparenchymally (Fig. 2). The drainage lymphatic vessel was found from injection point to the first IMLN (IM-SLN) after 10.5 \pm 0.35 minutes radiotracer injection and the image of the IM-SLN became more and more clearly which means radiotracer was accumulating into the IM-SLN. After that, the imaging of SPECT showed the second IMLN received the lymph drainage from the IM-SLN and then the third IMLN received the lymph drainage from the second IMLN. Thus, the lymphatic drai- nage pattern was step by step in the internal mammary area. The location of the IM-SLN was concentrated in the second $33.3 \%(5 / 15)$ and third $46.7 \%(7 / 15)$ intercostal space. Therefore, the IMLN lymphatic drainage is accord with the model as showing by Fig. 5. It demonstrated the accuracy of IM-SLN concept and the situation of IM-SLN could stand for the whole IMLN chain. This study provided more evidence to support that IM-SLN removed by IM-SLNB guiding with the modified radiotracer injection technique is true SLN in the IMLN chain. In our pilot study, we have demonstrated that the primary tumor area and the entire breast parenchyma could drain into the same IMLN [7]. Combined with the conclusion of the pilot study, we could conclude that IM-SLN receives lymphatic drainage from not only the primary tumor area, but also the entire breast parenchyma.

In conclusion, the lymphatic drainage pattern was presented by the small radiotracer $\left.{ }^{99 \mathrm{~m}} \mathrm{Tc}-D e x t r a n ~ 40\right)$ on the lymphoscintigraphy of SPECT/CT. In the IMLN chain, the lymph was identified draining from different regions of the breast to IM-SLN, and then outward from IM-SLN to other IMLN one by one. It demonstrated the concept of the IM-SLN and provided more evidences for the application of IM-SLNB in the clinical practice.

\section{Conflicts of Interest}

Conflict of interest relevant to this article was not reported.

\section{Acknowledgments}

The work was supported by grants from the National Natural Science Foundation of China $(81672638,81502314)$, the Natural Science Foundation of Shandong Province (2014ZRC03036), the Medical and Health Science Technology Development Program of Shandong Province (2017WS177), and the Science and Technology Planning Project of Shandong Academy of Medical Sciences (2016-39). YongSheng Wang and Xiao-Shan Cao conceived the study idea. Guo-Ren Yang conducted the literature searches and review of studies. YongSheng Wang and Xiao-Shan Cao performed data extraction, interpreted analyses. Yong-Sheng Wang advised on clinical content and on data resolution. Bin-Bin Cong and Peng-Fei Qiu provided additional data. Yong-Sheng Wang and Xiao-Shan Cao contributed to drafting the manuscript. Yong-Sheng Wang and Bin-Bin Cong contributed to reviewing the manuscript. We thank Yan-Bing Liu, MA, Tong Zhao, MA, Peng Chen, MA, Chun-Jian Wang, MA, and ZhaoPeng Zhang, MA from our breast cancer center. All six of these individuals contributed to manuscript review; none received compensation for their contributions. We thank Rogers W, PhD, an English teacher from University of Jinan, who contributed to revise the language of the manuscript. 


\section{References}

1. Connolly JL. Changes and problematic areas in interpretation of the AJCC Cancer Staging Manual, 6th Edition, for breast cancer. Arch Pathol Lab Med. 2006;130:287-91.

2. Gnerlich JL, Barreto-Andrade JC, Czechura T, John JR, Turk MA, Kennedy TJ, et al. Accurate staging with internal mammary chain sentinel node biopsy for breast cancer. Ann Surg Oncol. 2014;21:368-74.

3. Qiu PF, Liu YB, Wang YS. Internal mammary sentinel lymph node biopsy: abandon or persist? Onco Targets Ther. 2016;9: 3879-82.

4. Huang O, Wang L, Shen K, Lin H, Hu Z, Liu G, et al. Breast cancer subpopulation with high risk of internal mammary lymph nodes metastasis: analysis of 2,269 Chinese breast cancer patients treated with extended radical mastectomy. Breast Cancer Res Treat. 2008;107:379-87.

5. Thorsen LB, Offersen BV, Dano H, Berg M, Jensen I, Pedersen AN, et al. DBCG-IMN: a population-based cohort study on the effect of internal mammary node irradiation in early node-positive breast cancer. J Clin Oncol. 2016;34:314-20.

6. Whelan TJ, Olivotto IA, Parulekar WR, Ackerman I, Chua BH, Nabid A, et al. Regional nodal irradiation in early-stage breast cancer. N Engl J Med. 2015;373:307-16.

7. Cong BB, Qiu PF, Liu YB, Zhao T, Chen P, Cao XS, et al. Validation study for the hypothesis of internal mammary sentinel lymph node lymphatic drainage in breast cancer. Oncotarget. 2016;7:41996-2006.

8. Cong BB, Qiu PF, Wang YS. Internal mammary sentinel lymph node biopsy: minimally invasive staging and tailored internal mammary radiotherapy. Ann Surg Oncol. 2014;21:2119-21.

9. Lyman GH, Temin S, Edge SB, Newman LA, Turner RR, Weaver DL, et al. Sentinel lymph node biopsy for patients with early-stage breast cancer: American Society of Clinical Oncology clinical practice guideline update. J Clin Oncol. 2014;32:1365-83.

10. Schulze T, Mucke J, Markwardt J, Schlag PM, Bembenek A. Long-term morbidity of patients with early breast cancer after sentinel lymph node biopsy compared to axillary lymph node dissection. J Surg Oncol. 2006;93:109-19.

11. Kumar A, Puri R, Gadgil PV, Jatoi I. Sentinel lymph node biopsy in primary breast cancer: window to management of the axilla. World J Surg. 2012;36:1453-9.

12. Veronesi U, Marubini E, Mariani L, Valagussa P, Zucali R. The dissection of internal mammary nodes does not improve the survival of breast cancer patients. 30-year results of a randomised trial. Eur J Cancer. 1999;35:1320-5.

13. Byrd DR, Dunnwald LK, Mankoff DA, Anderson BO, Moe RE, Yeung RS, et al. Internal mammary lymph node drainage patterns in patients with breast cancer documented by breast lym- phoscintigraphy. Ann Surg Oncol. 2001;8:234-40.

14. Qiu PF, Cong BB, Zhao RR, Yang GR, Liu YB, Chen P, et al. Internal mammary sentinel lymph node biopsy with modified injection technique: high visualization rate and accurate staging. Medicine (Baltimore). 2015;94:e1790.

15. Turner-Warwick RT. The lymphatics of the breast. Br J Surg. 1959;46:574-82.

16. Cruikshank WC. The anatomy of the absorbing vessels of the human body. London: G. Nicol; 1786.

17. Sappey MP. Anatomie, Physiologie, Pathologie des vaisseaux Lymphatiques consideres chez L'homme at les Vertebres. Paris: A. Delahaye and E. Lecrosnier; 1874.

18. Pavlista D, Eliska O. Relationship between the lymphatic drainage of the breast and the upper extremity: a postmortem study. Ann Surg Oncol. 2012;19:3410-5.

19. Suami H, Pan WR, Mann GB, Taylor GI. The lymphatic anatomy of the breast and its implications for sentinel lymph node biopsy: a human cadaver study. Ann Surg Oncol. 2008; 15:863-71.

20. Tanis PJ, Nieweg OE, Valdes Olmos RA, Kroon BB. Anatomy and physiology of lymphatic drainage of the breast from the perspective of sentinel node biopsy. J Am Coll Surg. 2001;192: 399-409.

21. Yamaguchi A, Hanaoka H, Pirmettis I, Uehara T, Tsushima $Y$, Papadopoulos M, et al. Injection site radioactivity of $(99 \mathrm{~m})$ Tc-labeled mannosylated dextran for sentinel lymph node mapping. Mol Pharm. 2015;12:514-9.

22. Harrell MI, Iritani BM, Ruddell A. Lymph node mapping in the mouse. J Immunol Methods. 2008;332:170-4.

23. Mariani G, Erba P, Villa G, Gipponi M, Manca G, Boni G, et al. Lymphoscintigraphic and intraoperative detection of the sentinel lymph node in breast cancer patients: the nuclear medicine perspective. J Surg Oncol. 2004;85:112-22.

24. Kraft O, Havel M. Sentinel lymph nodes and planar scintigraphy and SPECT/CT in various types of tumours. Estimation of some factors influencing detection success. Nucl Med Rev Cent East Eur. 2013;16:17-25.

25. Brouwer OR, Vermeeren L, van der Ploeg IM, Valdes Olmos RA, Loo CE, Pereira-Bouda LM, et al. Lymphoscintigraphy and SPECT/CT in multicentric and multifocal breast cancer: does each tumour have a separate drainage pattern? Results of a Dutch multicentre study (MULTISENT). Eur J Nucl Med Mol Imaging. 2012;39:1137-43.

26. Vercellino L, Ohnona J, Groheux D, Slama A, Colletti PM, Chondrogiannis S, et al. Role of SPECT /CT in sentinel lymph node detection in patients with breast cancer. Clin Nucl Med. 2014;39:431-6. 